\title{
Evaluation de la pertinence des projets et programmes de développement rural au Cameroun
}

\section{Awé Baïna Modeste,}

Doctorant, Département de Socio-économie Rurale et de Vulgarisation agricole. Faculté d'Agronomie et des Sciences Agricoles,

Université de Dschang

\section{Maître de Conférence Tankou Christopher Mubuteneh,}

Chargé de cours, Département d'Agriculture.

Faculté d'Agronomie et des Sciences Agricoles, Université de Dschang Maître de Conférence Manu Ibrahim Nformi,

Chargé de cours, Département de Socio-économie Rurale et de Vulgarisation agricole. Faculté d'Agronomie et des Sciences Agricoles,

Université de Dschang

Doi:10.19044/esj.2020.v16n29p105 URL:http://dx.doi.org/10.19044/esj.2020.v16n29p105

\section{Résumé}

Cette étude portant sur l'évaluation de la pertinence des projets et programmes de développement rural au Cameroun, a été réalisée en 2019. Son objectif global était de contribuer à l'appréciation de la pertinence des projets et programmes développement rural au Cameroun. Les données ont été collectées avec un guide d'entretien et des trames d'enquête lors des entretiens individuels et de groupes avec les différentes parties prenantes des projets et programmes de développement rural au Cameroun. Un échantillon de trois cent soixante-neuf (369) personnes ont été enquêtées avec un taux de participation de $91,86 \%$. Les statistiques descriptives ont été utilisées pour l'analyse des données avec les logiciels SPSS.26 et Excel. Pour d'autres types de données, leur cohérence a été vérifiée par comparaison à la littérature existante. Il ressort de l'analyse que : les objectifs de ces projets et programmes de développement rural sont en cohérence (alignement à 75\%) avec les objectifs stratégiques des partenaires techniques et financiers ainsi que du Gouvernement. Les objectifs des projets et programmes de développement rural sont à $80 \%$ trop ambitieux, à $16 \%$ ambitieux et seulement à $2 \%$ peu ambitieux par rapport aux résultats de leurs mises en œuvre. Leur ciblage social est en adéquation avec les besoins d'appuis à $86 \%$, par contre, $14 \%$ estiment que le ciblage n'est pas en adéquation. La pertinence globale moyenne des projets et programmes de développement rural au Cameroun est jugée modérément insatisfaisante. Il serait nécessaire, voir impératif, de 
repenser les stratégies et les approches de conception et de mise en œuvre des projets et programmes de développement rural au Cameroun.

Mots clés : Evaluation, Pertinence, Projet, Programme, Développement rural

\title{
Relevance evaluation of rural development projects and programs in Cameroon Awé Baïna Modeste
}

\author{
Awé Baïna Modeste, \\ Doctorant, Département de Socio-économie Rurale et de Vulgarisation \\ agricole. Faculté d'Agronomie et des Sciences Agricoles, \\ Université de Dschang \\ Maître de Conférence Tankou Christopher Mubuteneh, \\ Chargé de cours, Département d'Agriculture. \\ Faculté d'Agronomie et des Sciences Agricoles, Université de Dschang \\ Maître de Conférence Manu Ibrahim Nformi, \\ Chargé de cours, Département de Socio-économie Rurale et de Vulgarisation \\ agricole. Faculté d'Agronomie et des Sciences Agricoles, \\ Université de Dschang
}

\begin{abstract}
This study on the evaluation of the relevance of rural development projects and programs in Cameroon was carried out in 2019. Its overall objective was to contribute to the assessment of the relevance of rural development projects and programs in Cameroon. The data was collected with an interview guide and survey frames during individual and group interviews with the various stakeholders of rural development projects and programs in Cameroon. A sample of three hundred and sixty-nine (369) people were surveyed with a participation rate of $91.86 \%$. Descriptive statistics were used for data analysis with SPSS.26 and Excel software. For other types of data, their consistency was checked by comparison with the existing literature. The analysis shows that: the objectives of these rural development projects and programs are consistent (75\% alignment) with the strategic objectives of the technical and financial partners as well as the Government. The objectives of rural development projects and programs are 80\% too ambitious, 16\% ambitious and only $2 \%$ not very ambitious in relation to the results of their implementation. $86 \%$ of their social targeting is in line with support needs, on the other hand, $14 \%$ believe that the targeting is not in line. The overall
\end{abstract}


average relevance of rural development projects and programs in Cameroon is rated moderately unsatisfactory. It would be necessary, if not imperative, to rethink the strategies and approaches for the design and implementation of rural development projects and programs in Cameroon.

Keywords: Evaluation, Relevance, Project, Program, Rural development

\section{Introduction}

\subsection{Contexte}

Dans toutes les régions du monde, les taux d'extrême pauvreté et d'insécurité alimentaire sont plus élevés dans les zones rurales où, selon les estimations, vivent trois quarts des personnes extrêmement pauvres et souffrant d'insécurité alimentaire dans le monde (FIDA, 2017). La Banque Mondiale (2007) précise que, les trois quarts des habitants pauvres des pays en développement vivent dans les espaces ruraux, et la plupart d'entre eux tirent, directement ou indirectement, leur subsistance de l'agriculture. L'agriculture est déterminante parce que $75 \%$ des pauvres du monde vivent dans les zones rurales, et parce que ce secteur a une grande influence sur la croissance économique, la réduction de la pauvreté et la viabilité à long terme de l'environnement (Banque Mondiale, 2008).

La proportion de la population vivant en-dessous du seuil de pauvreté au Cameroun est passée de 40,2\% en 2001 à 37,5\% en 2014 enregistrant une baisse de 2,7\% sur la période. Bien loin de la cible qui était de situer le taux de pauvreté à $25,3 \%$ en 2015 . La cible visée reste très éloignée du niveau de performance dans ce domaine 15 ans après. Cette pauvreté demeure un phénomène prépondérant en milieu rural et ce, malgré tous les efforts déployés à travers les politiques de développement. Contrairement au milieu urbain où on connait une baisse du taux de pauvreté de $9,0 \%$ entre 2001 et 2014, il a plutôt augmenté en milieu rural, passant de $52,1 \%$ à $56,8 \%$ sur la même période, (INS, 2015).

La Banque Mondiale (2008) estime que dans la majorité des pays d'Afrique subsaharienne, le secteur agricole constitue 29\% du PIB en moyenne, la pauvreté touche $80 \%$ de la population totale, dont $70 \%$ des ruraux. L'agriculture et les secteurs qui lui sont associés sont essentiels pour le développement mais aussi pour la lutte contre la pauvreté de masse et l'insécurité alimentaire, car ils sont source d'emplois, d'activité et de revenus dans ces pays. Par ailleurs, l'analyse des contributions sectorielles au PIB au Cameroun met en exergue les gains probables liés à la diversification de l'économie, l'accent étant mis sur la relance de l'agriculture, la transformation des matières premières et le lancement de grands projets d'infrastructures. Considéré comme prioritaire, le secteur primaire connaîtrait une croissance de 
$5 \%$ en moyenne annuelle sur la période $2010-2020$ avec un pic de 5,5\% en 2015, (DSCE, 2010).

Les projets de développement rural sont des opportunités créées dans le but de répondre à des besoins fondamentaux, spécifiques et vitaux des populations socioéconomiquement les plus démunies. D'une manière générale, ils constituent sans conteste un moyen mis au profit des groupes sociaux en difficulté pour se rattraper et participer au développement local, approche du développement en vogue depuis les années 70 (Fontil, 2009).

\subsection{Problématique}

Ela (1990) évoque les nombreux échecs des projets de développement qui laissent la place à l'éclosion de relations conflictuelles entre dirigeants et paysans. Il met l'accent sur les sommes faramineuses investies pour développer l'Afrique mais qui finissent par n'avoir que des conséquences néfastes perçues à travers l'éclatement des groupes sociaux, les conflits d'intérêts, etc. puisque des populations vivant sur un même territoire sont appelées à défendre des intérêts de natures diverses. D'après une étude parue en 2005, dans le compte de la Banque Française de Développement, il en ressort que toute l'aide publique apportée aux pays de la zone franc n'a pas créé autant de richesses dans ces pays que les revenus privés directement investis la plupart du temps par les ressortissants de ces pays en France. La raison évoquée pour justifier cet état de fait est essentiellement l'inadéquation entre les prestations proposées et les problèmes réels des populations. Merlin (1991) en parle quand il souscrit à la thèse selon laquelle une aide, pour être bénéfique, ne doit venir qu'en réponse à un désir manifeste par le bénéficiaire.

Suite à ces multiples échecs enregistrés dans la conception et la mise en œuvre des projets et des programmes de développement rural, de nouvelles méthodes dites participatives, rapides et interactives ont été développées vers le début des années 80. Selon l'OCDE et FAO (2016) entre 1973 et 2004, l'aide multilatérale reçue par le Cameroun en trente ans s'est élevée à environ 600 milliards de francs CFA. Toutefois, la volatilité de l'aide n'est pas favorable au développement d'actions structurelles porteuses. Dans le domaine du développement rural un point d'attention doit être mis sur l'évaluation.

Pour apprécier le niveau d'atteinte de ces objectifs des divers projets et programmes, il faut analyser leur pertinence. D'où la préoccupation de la présente étude qui s'interroge sur la pertinence des projets et programmes de développement rural au Cameroun. Ceci suscite un questionnement en rapport avec l'organisation de l'intervention des Projets et des Programmes de développement rural au Cameroun. Quel est la pertinence des projets et programmes pour le développement rural au Cameroun ? Dans le souci d'apporter des réponses à la fois constructives et réalistes à la problématique, 
il s'avère indispensable de savoir si : les projets et programmes de développement rural sont conçus et mis en œuvre de manière pertinente ? Cet article s'articule autour de l'introduction, la méthodologie, les résultats et discussions ainsi que la conclusion.

\section{Objectifs de l'étude}

La présente étude se propose de contribuer à l'amélioration des connaissances sur l'évaluation de pertinence des projets et programmes de développement rural au Cameroun. L'atteinte de cet objectif global nécessite la réalisation des objectifs spécifiques ci-après :

1) Evaluer la pertinence de la conception et de la mise en œuvre des projets et programmes de développement rural au Cameroun ;

2) Evaluer la plus-value des projets et programmes de développement rural au Cameroun.

\section{METHODOLOGIE DE LA RECHERCHE}

Cette section présente en détails : le choix et la présentation de la zone d'étude, le choix de la méthode, les types de données, les outils d'enquête, les populations d'étude, les échantillonnages, les variables et l'analyse des données en rapport avec nos travaux.

\subsection{Choix de la zone d'étude}

L'étude a été menée au Cameroun parce que la problématique du développement rural via les Projets et les Programmes est le cheval de bataille depuis plusieurs décennies.

\subsection{Présentation de la zone d'étude}


Figure 2 : Carte des zones agroécologiques du Cameroun

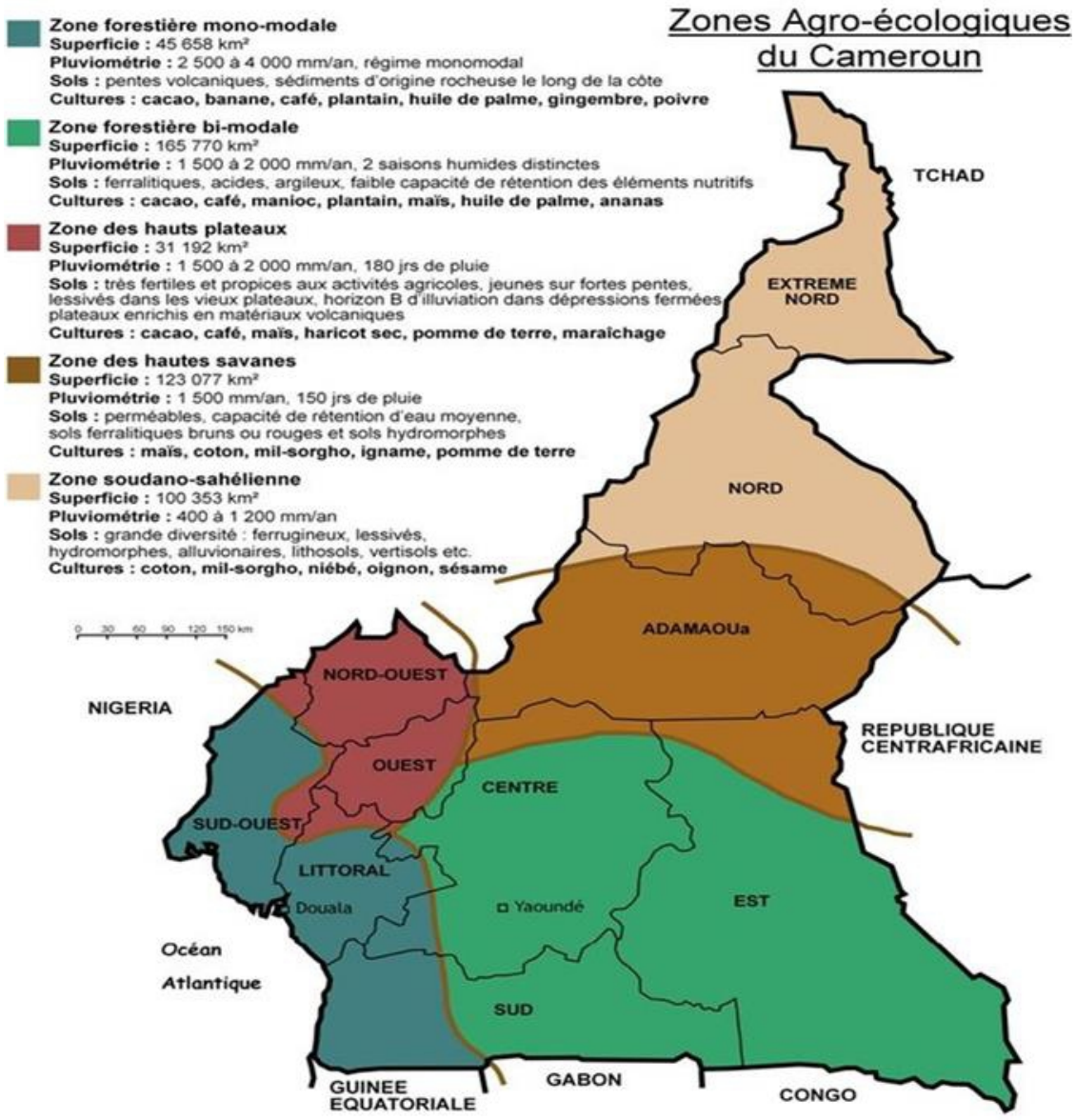

Source : Annuaire statistique du Cameroun, 2000

L'agriculture est très présente au Cameroun. Tout comme l'environnement, l'agriculture est très diversifiée. Selon la Banque Mondiale (2007), elle peut être considérée comme le pilier de l'économie du Cameroun avec une contribution de $20 \%$ au PIB. En 2009, il ressort que $42 \%$ de la population est rurale et la croissance de l'agriculture est de $4 \%$ par an depuis 1988 (Banque Mondiale, 2008).

\subsubsection{Collecte des données}

Pour mener à bien cette recherche, la collecte des données s'est faite à l'aide de la recherche documentaire et des entretiens semi-directifs.

Ces données ont été collectées aux moyens des trames d'enquêtes (questionnaires), des guides d'entretien et des observations directes sur le terrain. Durant cette phase, des entretiens ont eu lieu avec des personnes ressources : de la Direction d'Etudes, Planification et Coopération (DEPC) ainsi que de la cellule de suivi du MINADER, de la Direction d'Etudes, 
Planification, Coopération et Statistique (DEPCS) ainsi que de la cellule de suivi du MINEPIA et l'équipe de gestion et du suivi du Development Assistance Database (DAD) du Ministre de l'Economie, de la Planification et de l'Aménagement du Territoire (MINEPAT), des représentants des agences d'appui technique et financier, des Coordonnateurs de projets et programmes, des responsables de suivi-évaluation, des partenaires techniques stratégique et opérationnels, des bénéficiaires et des populations riveraines.

Par ailleurs, une revue de la documentation disponible dans les archives des ministères de tutelles, des différents partenaires techniques et financiers et des Projets/programmes de développement rural en lien avec les évaluations ont complété les données collectées.

Les enquêtes ont eu lieux avec des trames d'enquêtes bien structurées et normatives.

\subsubsection{Méthode d'échantillonnage}

L'échantillon était de type probabiliste et la technique d'échantillonnage par grappes a été utilisée. Il s'agissait de subdiviser une population homogène (projets/programmes de développement rural) en grappe (agence d'appui technique et financier ou bailleur de fonds).

Le type d'échantillonnage utilisé lors des entretiens est la méthode « boule de neige ». Cette méthode consiste à identifier dans un premier temps quelques acteurs qui interviennent dans le développement rural et qui sont connus. A partir de ces derniers, d'autres acteurs additionnels ont été recensés ; ce, jusqu'à ce qu'il n'y ait plus d'information nouvelle.

Le choix des enquêtés s'est fait sur la base du critère d'opérationnalité lors des entretiens. Ce critère a permis de recenser des acteurs de développement rural sur l'ensemble du territoire. Les différents acteurs recensés sur la base de ce critère ont constitué l'échantillon.

Un échantillon raisonné a été constitué de façon aléatoire et soumis à l'enquête. Le groupe proposé était composé de trois cent soixante-neuf (369) individus qui ont constitué l'échantillon avec un taux de participation effectif à l'enquête et aux entretiens de 91,86\%, soit trois cent trente-neuf (339).

La taille d'échantillon s'est calculée avec la formule suivante :

- $\mathrm{n}=\mathrm{t}^{2} \times \mathrm{p} \times(1-\mathrm{p}) / \mathrm{m}^{2}$;

- $\mathrm{n}$ : Taille d'échantillon minimale pour l'obtention de résultats significatifs pour un événement et un niveau de risque fixé ;

- $\quad \mathrm{t}$ : Niveau de confiance (la valeur type du niveau de confiance de $95 \%$ sera 1,96);

- $\quad \mathrm{p}$ : proportion estimée de la population qui présente la caractéristique ;

- m : Marge d'erreur (généralement fixée à $5 \%$ ).

Ainsi, pour un événement ayant une probabilité de réalisation de $40 \%$, en prenant un niveau de confiance de $95 \%$ et une marge d'erreur de $5 \%$, la 
taille d'échantillon devra être de $: \mathrm{n}=1,96^{2} \times 0,4 \times 0,6 / 0,05^{2}=368,79$. Soit 369 individus.

\subsubsection{Variables}

La recherche quantitative a pour objet de tester la validité du modèle de recherche. L'étude a été composée de deux types de variables. Dans ces deux types de variables, des variables indépendantes (les éléments d'évaluation des projets de développement rural) et une variable dépendante (pertinence des projets et programmes de développement rural). La deuxième est la variable expliquée ou dépendante, c'est celle dont on recherchait la réponse. Ici, c'était « la pertinence des projets et programmes ». La variable explicative, la première, est la cause du phénomène à expliquer.

\subsection{Analyse des données}

Cette section porte sur la manière dont s'est réalisée l'analyse, les techniques statistiques qui ont été employées, l'utilisation des programmes informatiques, etc. Les instruments de mesure peuvent être définis comme les supports matériels nécessaires à la collecte des données d'étude ; dans la plupart des cas, il s'agit du questionnaire et de guide d'enquête. Dans le cadre de notre étude, le questionnaire a été retenu comme instrument de mesure compléter par un guide d'entretien. Dans le cadre de cette étude, les logiciels SPSS version 26 et Excel ont été utilisé pour la pertinence de leurs résultats, leur simplicité, leur grand nombre de procédures statistiques. Ces logiciels nous ont permis de faire les statistiques descriptives (fréquence, moyenne entre autres) qui ont permis d'analyser certaines données. Pour les données issues des entretiens semi-directifs, des croisements et des tris des données/informations nous ont permis de faire le cadrage sémantique des résultats. Cette méthode de croisement et de tri consistait à regrouper les mêmes données par thème, faire des croisements entre les différents thèmes sur la base des modèles proposés pour leur mise en place et les comparer avec la littérature existante.

Pour analyser la relation entre les variables, le coefficient de corrélation linéaire de Pearson (r), qui examine à la fois la puissance et la direction de la relation linéaire qui existe entre deux variables continues a été faite. Pour cette analyse, un coefficient compris entre 0,8 et 1 , signifie qu'il y a une corrélation forte entre les deux variables qui diminuent ou augmentent ensemble selon le signe de la valeur du coefficient de corrélation. Un coefficient compris entre 0,5 et 0,799 signifie qu'il existe une relation modérée entre les deux variables qui diminuent ou augmentent ensemble selon le signe de la valeur du coefficient de corrélation. Un coefficient inférieur à $-0,5$ ou 0,5 signifie que la corrélation est non significative c'est-à-dire pas de corrélation 
entre les deux variables qui diminuent ou augmentent ensemble selon le signe de la valeur du coefficient de corrélation.

\section{RESULTATS ET DISCUSSIONS}

Dans le cadre de cette étude, la pertinence des projets et programmes de développement rural au Cameroun est évaluée à l'aide des critères de notation établis sur une échelle de six points qui sont les suivants : très satisfaisant (6), satisfaisant (5), modérément satisfaisant (4), modérément insatisfaisant (3), insatisfaisant (2) et très insatisfaisant (1). La pertinence examine le bien-fondé du projet/programme au regard de ses objectifs et de ses enjeux. Elle est la mesure pour laquelle les objectifs du projet correspondent aux attentes des bénéficiaires et aux besoins du pays. La pertinence d'un projet/programme est principalement influencée par sa conception et sa mise en œuvre. Elle mesure la justesse existante entre les objectifs envisagés par le projet et les problèmes identifiés ou les besoins réels. La pertinence doit être évaluée tout au long du cycle de vie du projet.

Les éléments qui peuvent être examinés pour la détermination de la pertinence sont :

$>$ La concordance avec les finalités des stratégies nationales de développement rural ;

$>$ La correspondance entre les objectifs et les besoins et/ou les attentes des populations bénéficiaires ;

$>$ La conformité avec les orientations générales du secteur rural.

Une action est dite pertinente si ses objectifs explicites sont en adéquation avec la nature des problèmes qu'elle est censée résoudre ou prendre en charge.

La présentation de la zone d'étude est extraite de l'Annuaire Statistique du Cameroun de l'INS, (2010). Le Cameroun est situé au fond du golfe de Guinée, entre le deuxième et le douzième degré Nord dans le golfe de Guinée, le Cameroun se présente comme un grand triangle s'inscrivant entre l'Océan Atlantique, le lac Tchad et le bassin du Congo. Il a des frontières avec le Nigeria, le Tchad, la République Centrafricaine, le Congo, le Gabon et la Guinée équatoriale. Le Cameroun est un territoire vaste d'une superficie totale de $475650 \mathrm{~km} 2$, dont $466.050 \mathrm{~km} 2$ pour la partie continentale et $9600 \mathrm{~km} 2$ pour la partie maritime. Le taux de croissance démographique est d'environ 2,8\% par an. 56\% de la population à moins de 20 ans.

Le pays est organisé en 10 régions découpées en 58 départements et 373 arrondissements. Le processus de décentralisation en cours permettra à terme le transfert de certaines compétences administratives et financières de l'État vers la Commune qui épouse en général le contour géographique d'un arrondissement. Le pays comporte une variété de paysages, de zones géomorphologiques et climatiques qui peuvent être regroupées en cinq entités 
régionales ou zones agroécologiques distinctes. Du point de vue de l'étendue et des caractéristiques pluviométriques et pédologiques (qui sont parmi les plus importantes pour la production agricole), les cinq grandes unités écologiques qui composent le territoire national sont : (i) la zone soudano sahélienne, (ii) la zone de savane guinéenne, (iii) la zone des hauts plateaux de l'Ouest, (iv) la zone côtière et maritime et, (v) la zone des forêts tropicales (voir figure 1).

\subsection{Evaluation de l'alignement des projets et programmes aux préoccupations stratégiques des parties prenantes}

Tableau 2 : Analyse de l'alignement des objectifs des projets et programmes de développement rural aux objectifs stratégiques des PTF et du Gouvernement

\begin{tabular}{|l|c|c|}
\hline \multicolumn{1}{|c|}{ Désignation } & Nombre & Fréquence (\%) \\
\hline $\begin{array}{l}\text { Objectifs globaux des projets et programmes de } \\
\text { développement rural aligné avec les objectifs } \\
\text { stratégiques des PTF et du secteur rural du } \\
\text { Gouvernement }\end{array}$ & 3 & 75 \\
\hline $\begin{array}{l}\text { Objectifs globaux des projets et programmes de } \\
\text { développement rural non alignés avec les objectifs } \\
\text { stratégiques des PTF et du secteur rural du } \\
\text { Gouvernement }\end{array}$ & 1 & 25 \\
\hline \multicolumn{1}{|c|}{ TOTAL } & $\mathbf{5}$ & $\mathbf{1 0 0}$ \\
\hline
\end{tabular}

L'analyse du tableau 2 montre que les objectifs des projets et programmes de développement rural selon leurs documents de conception sont en cohérence (alignement à 75\%) avec les objectifs stratégiques de développement du secteur rural des PTF et du Gouvernement. Cela peut s'expliquer par le fait que les objectifs des projets et programmes de développement rural intègrent les préoccupations des différentes parties prenantes lors de leurs conception, d'où leurs pertinences satisfaisantes.

Les objectifs des projets et programmes de développement rural évalués sont en concordance avec les objectifs stratégiques du secteur rural au Cameroun, depuis les deux dernières décennies correspondant à la période d'évaluation par rapport à leur alignement à hauteur de $75 \%$ aux objectifs stratégiques des parties prenantes. Les objectifs stratégiques montrent que l'ensemble des objectifs spécifiques des projets devaient contribuer à l'atteinte d'au moins un objectif stratégique de développement du secteur rural au pays. Une exception est à faire pour l'objectif lié aux infrastructures sociales en milieu rural qui n'est aligné à aucun objectif stratégique du pays, mais qui peut cependant être relié directement à l'amélioration des conditions de vie en milieu rural. Nous notons aussi que les objectifs spécifiques des projets et programmes sont alignés avec des stratégies pays de manière consécutive et continue. 


\subsection{Appréciation des objectifs et des approches des projets et programmes par les différents acteurs}

Tableau 3 : appréciation faite par les différentes parties prenantes sur l'alignement des objectifs des projets et programmes de développement rural aux objectifs stratégiques du secteur rural des PTF et du Gouvernement

\begin{tabular}{|c|c|c|}
\hline Appréciation & Effectif & Fréquence (\%) \\
\hline Très utile & 311 & 92 \\
\hline Utile & 27 & 8 \\
\hline Peu utile & 0 & 0 \\
\hline Inutile & 0 & 0 \\
\hline TOTAL & $\mathbf{3 3 8}$ & $\mathbf{1 0 0}$ \\
\hline
\end{tabular}

Les résultats du tableau 3 montrent que les répondants estiment que les objectifs et priorités des projets et programmes sont d'une façon générale utile, très utiles à $92 \%$ et utiles à $8 \%$. Ce qui veut dire que les objectifs définis des projets et programmes correspondent aux attentes des acteurs. Le bien-fondé des objectifs des projets et programmes selon les différents acteurs, réside dans son arrimage ou alignement aux objectifs stratégiques du secteur rural des PTF et du Gouvernement.

Les objectifs des projets et/ou programmes des partenaires techniques et financiers, les politiques et stratégies du Gouvernement liées au secteur agricole et rural ont quatre grands objectifs en commun. Ces objectifs en commun sont : i) la réduction de la pauvreté rurale par l'amélioration des infrastructures en milieu rural, l'augmentation des revenus des populations rurales, l'amélioration des conditions sanitaires, l'amélioration de la scolarité en milieu rural etc.; ii) la sécurité alimentaire par l'amélioration de la productivité agropastorale et du statut nutritionnel ; iii) la gestion durable des ressources naturelles et ; iv) l'emploi des jeunes ruraux par la promotion de l'auto emploi et l'amélioration des qualifications professionnelles des jeunes ruraux pour faciliter leur insertion en milieux professionnels (DSCE, 2010). Les projets et programmes des partenaires techniques et financiers étant bien alignés et en cohérence avec les politiques et stratégies du Gouvernement camerounais, ils sont donc aussi globalement conformes aux politiques, aux stratégies et aux priorités Gouvernementales.

Par ailleurs, Ahizi (2019) estime que, les bailleurs de fonds et les pays récipiendaires sont confrontés à un défi majeur : accroitre la pertinence des projets/programmes qui se caractérise par l'atteinte d'objectifs bien ciblés et déterminés en fonction des stratégies nationales définies par les pays bénéficiaires. 


\subsection{Evaluation de l'appréciation des objectifs et des approches des projets et programmes par les différents acteurs}

Tableau 4 : Appréciation des différentes approches ou stratégies de mises en œuvre des projets et programmes de développement rural par les parties prenantes

\begin{tabular}{|c|c|c|c|c|c|c|c|c|c|c|c|c|c|c|}
\hline \multirow{2}{*}{ 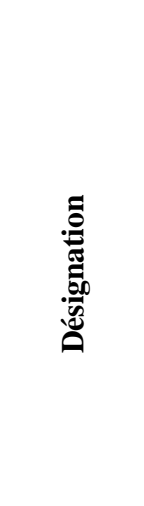 } & \multicolumn{2}{|c|}{ 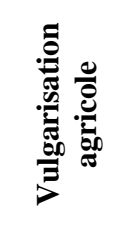 } & \multicolumn{2}{|c|}{ 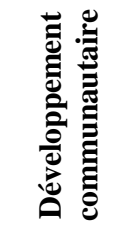 } & \multicolumn{2}{|c|}{ 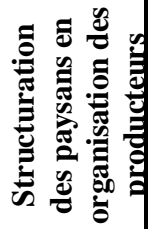 } & \multicolumn{2}{|c|}{ 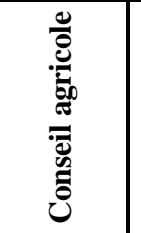 } & \multicolumn{2}{|c|}{ 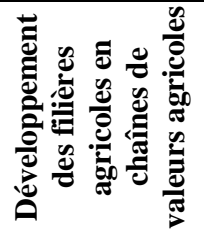 } & \multicolumn{2}{|c|}{ 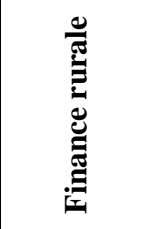 } & \multicolumn{2}{|c|}{ 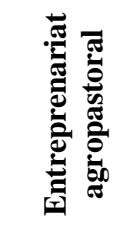 } \\
\hline & 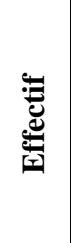 & 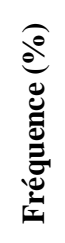 & 胥 & 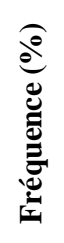 & 莺 & 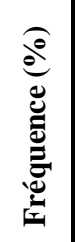 & 莺 & 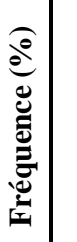 & 胥 & 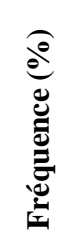 & 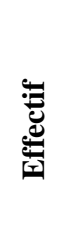 & 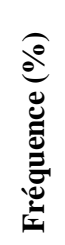 & 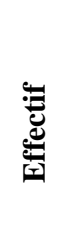 & 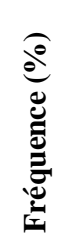 \\
\hline Très utile & 305 & 90 & 299 & 88 & 294 & 87 & 302 & 89 & 223 & 66 & 236 & 70 & 279 & 83 \\
\hline Utile & 32 & 9 & 26 & 8 & 23 & 7 & 22 & 7 & 62 & 18 & 67 & 20 & 36 & 11 \\
\hline Peu utile & 1 & 0 & 9 & 3 & 15 & 4 & 14 & 4 & 37 & 11 & 23 & 7 & 14 & 4 \\
\hline Inutile & 0 & 0 & 4 & 1 & 6 & 2 & 0 & 0 & 16 & 5 & 12 & 4 & 9 & 3 \\
\hline TOTAL & 338 & 100 & 338 & 100 & 338 & 100 & 338 & 100 & 338 & 100 & 338 & 100 & 338 & 100 \\
\hline
\end{tabular}

L'analyse du tableau 4 montre que les répondants estiment que les approches sont très utiles de $66 \%$ à $90 \%$, utiles de $7 \%$ à $20 \%$, avec une marge faible des appréciations peu utile de $0 \%$ à $11 \%$ et inutile de $0 \%$ à $5 \%$. Ce qui veut dire que les objectifs des projets et programmes de développement rural sont perçus presque de la même manière par tous les acteurs. Le bien-fondé des projets et programmes de développement rural selon les différents acteurs réside dans son arrimage ou alignement aux stratégies des PTF et du Gouvernement en rapport avec le secteur rural.

Au cours des dix-huit dernières années, les partenaires techniques et financiers à travers les projets et les programmes ont mis en œuvre au moins sept approches de développement distinctes devant permettre d'atteindre les objectifs stratégiques nationales. Il s'agit entre autres de : i) la vulgarisation agricole, ii) le développement communautaire ; iii) la structuration des paysans en organisation des producteurs ; iv) le conseil agricole, v) le développement des filières agricoles en chaînes de valeurs agricoles ; vi) la finance rurale ; vii) l'entreprenariat agropastoral. L'évolution des approches était en ligne avec les évolutions du cadre stratégique national et international. Ces approches avaient du sens à la conception du fait de la prise en compte 
des besoins identifiés des ruraux pauvres au Cameroun et de la durabilité de leur impact.

Toutefois, ces approches ont été trop simplifiées ou mal adaptées pour les raisons suivantes: analyse trop superficielle de la réalité ou la faible maîtrise de l'approche par les équipes en charge de la mise en œuvre ; du besoin de rattraper les retards de mise en œuvre (raccourcis) ; ou de se conformer aux exigences des partenaires techniques et financiers ou du Gouvernement ; ou encore par manque de capacité technique des équipes des projets/programmes ou prestataires de services face à la complexité globale des projets et programmes. Ces modifications ont réduit l'efficacité des approches et donné lieu à des résultats peu durables. Pour y arriver, OCDE, (2005 et 2008), montrent que ceux-ci ont pris un certain nombre d'engagements à savoir : 1) Avoir une gestion axée sur les résultats (GAR), c'est-à-dire un mode de gestion qui établisse un lien entre la dépense publique et l'atteinte d'un résultat concret ;2) Et, harmoniser un cadre d'évaluation de la performance.

Cependant, les différents fonds que les projets et programmes devaient mettre en place ont été soit non opérationnalisés, soit gérés directement par les unités de Coordination ou Gestion des projets/programmes, alors qu'ils devaient être gérés avec une forte implication des bénéficiaires directs. Selon la FAO, (2014), c'est pour cela qu'un pas de plus a été fait vers une harmonisation accrue des priorités avec le Programme détaillé pour le développement de l'agriculture africaine (PDDAA). Certains donateurs, au lieu de financer des projets et des programmes indépendants, préfèrent désormais apporter un soutien budgétaire ou un financement basé sur les politiques.

Les projets et programmes de développement rural ont surtout promues les productions agropastorales et une vulgarisation de nouvelles pratiques culturales. La moitié des bénéficiaires enquêtés ont décrit un changement qui concerne principalement les questions liées à l'augmentation de production. Selon Pasha, (2002), les interventions de développement dans les secteurs agricole et para-agricole visent toutes, à sortir les producteurs ruraux de la précarité en leur assurant un meilleur revenu et/ou la sécurité alimentaire. Ainsi, suivant leurs approches et conceptions de la notion de pauvreté, les actions de ces projets/programmes ont été globalement sous-tendues par la logique d'augmentation des productions agricoles en jouant sur la productivité agricole et son accroissement durable. D’après Yécy, (2016), de tous ces domaines d'attention des projets/programmes, ceux se rapportant à l'agriculture concentrent à eux seuls plus de 75\% des interventions. Ils visent à moderniser et à lutter contre la pauvreté en milieu rural. Dans ce cadre, des actions de soutien et de promotion à la production (appui en intrants, amélioration d'itinéraires techniques, gestion et amélioration de la fertilité des 
sols, soutien à la mécanisation, etc.) ont été menées par les projets et programmes de développement rural. Toutefois, l'aspect "filière" était largement limité à un appui à la commercialisation à travers la subvention d'infrastructures telles que la construction des marchés ruraux, la construction des magasins de stockage et quelques petits équipements de transformation des produits agricoles avec une très faible implication des bénéficiaires dans les chantiers de construction. Par ailleurs, ces projets n'ont pas suffisamment pris en compte l'analyse économique dans leurs appuis aux organisations des producteurs.

La plupart des projets et programmes ne prennent pas en compte l'importance de la diversification des activités comme stratégie de gestion des risques et d'optimisation de l'utilisation des ressources (main d'œuvre, trésorerie, terre etc.) des producteurs. Suivant leurs orientations et objectifs, les projets et programmes, appuient des producteurs ou des organisations des producteurs spécialisés (Groupe ment d'Intérêt Economique, coopératives, etc.) dans un seul maillon d'une spéculation au lieu de s'intéresser à de nombreuses activités économiques intégrées dans la chaine de valeur de la spéculation. En réalité, les organisations des producteurs et les producteurs ruraux sont rarement spécialisées dans une seule activité économique. Le développement du secteur agropastoral devrait davantage prendre en compte la diversité des activités économiques en leur sein, et apporter des outils de gestion des ressources dans le temps et dans l'espace pour optimiser leur utilisation.

\subsection{Evaluation de la prise en compte des préoccupations des différentes parties prenantes lors de la conception des projets et programmes de développement rural}

Tableau 5 : Analyse de la portée des objectifs des projets et programmes de développement rural par rapport à leurs mises en œuvre

\begin{tabular}{|l|c|c|}
\hline Portée & Nombre & Fréquence (\%) \\
\hline Trop ambitieux & 45 & 80 \\
\hline Ambitieux & 9 & 16 \\
\hline Peu ambitieux & 2 & 4 \\
\hline TOTAL & 56 & 100 \\
\hline
\end{tabular}

Il ressort du tableau 5 que les objectifs des projets et programmes de développement rural sont à $80 \%$ trop ambitieux, à $16 \%$ ambitieux et seulement à $2 \%$ peu ambitieux par rapport aux réalités de leurs mises en œuvre. En général, les projets et programmes de développement rural prennent en compte, les préoccupations des différentes parties prenantes dans leurs conceptions. Toutefois, ils ont des objectifs trop ambitieux vis-à-vis des réalités de leur mise en œuvre. 
L'ensemble des projets et programmes du portefeuille des partenaires techniques et financiers, à peu d'exception près, ont une conception trop ambitieuse et complexe par rapport aux capacités des unités de gestion desdits projets/programmes et des autres parties prenantes tels que : des prestataires de services. Aussi, la capacité des missions de supervision de ces projets et programmes chargées à bien orienter les responsables pour l'amélioration de leur performance revêt souvent bien des défaillances. Dans l'ensemble, les objectifs et cibles fixés par les différents projets et programmes ont été trop ambitieux par aux vues des réalités liées aux capacités locales, à la capacité de consommation des moyens financiers disponibles, aux procédures de passation des marchés et au temps disponible pour la mise en œuvre des projets et programmes. Dans $90 \%$ des cas, les documents de conception des projets et programmes ne prennent pas suffisamment en compte les mesures de capitalisation des acquis et de pérennisation des actions mises en œuvre par projet/programme au cours de la phase post projet/programme.

Selon Dey, (1984), cette inadéquation des interventions et de leurs cibles est due à la faible participation des populations rurales (la cible) dans la conception des projets et programmes ruraux ainsi que l'absence de formation particulière du personnel des projets et programmes sur les thématiques telles que l'écoute active des bénéficiaires. La FAO (2014) corrobore cette affirmation et estime que pour presque tous les projets $d$ 'investissement agricole, les donateurs se sont chargés de leur conception et de leur mise en œuvre sans impliquer les bénéficiaires ou les communautés à la base.

La conception des projets et programmes, ne tient pas suffisamment compte des réalités au niveau des bénéficiaires et de certaines parties prenantes. En effet, selon la FAO (2014), il transparait que les stratégies de croissance basées sur la production agricole définies de façon étroite et limitée, et imposées par les instances supérieures, n'avaient que peu à offrir à la plupart des petits et moyens producteurs dont les intérêts, priorités et capacités à adopter de nouvelles pratiques/nouvelles technologies sont trop hétérogènes. Les approches flexibles impulsées par la demande permettent de surmonter ces défis en proposant une grande variété d'innovations techniques possibles. $\mathrm{Ce}$, dans l'espoir d'offrir quelque chose à chaque partie et en dotant l'unité de gestion de projet des moyens nécessaires pour répondre aux demandes les plus diverses des clients.

Cette évolution en faveur des projets induits par la demande s'est accompagnée d'une tendance à confier au donateur la coordination à l'appui des programmes d'investissement dans le secteur agricole, au début, puis par des approches agricoles sectorielles. Tout en reflétant les pratiques des partenaires techniques et financiers de la conception des projets et programmes. Une approche complexe implique des défis de mise en œuvre très élevés et sous-entend de très longs processus de changement dans 
l'implémentation des documents de conception de projets et programmes. Il s'agit généralement du ciblage des plus pauvres avec une contribution des bénéficiaires prévue à hauteur de $0 \%$ à $50 \%$ selon les types d'appuis (les infrastructures sociales, les appuis aux activités génératrices de revenus, la mise en place de structures de décision locales, la création de groupements de producteurs, etc.). L'ensemble de ces projets et programmes travaillent avec de multiples partenaires dont les priorités divergentes et gèrent de nombreuses activités de différente nature, nécessitant des expertises diverses pour leur mise en œuvre. Ils opèrent avec des modalités d'intervention très différentes variant selon leur coordination ou l'approche d'intervention du partenaire technique et financier.

Par ailleurs, plusieurs projets ont été conçus avec l'espoir qu'ils pouvaient être bâtis sur des résultats d'autres projets existants. Ainsi, la conception de ces autres projets et programmes ne prévoyaient pas certaines activités supposées être prises en compte par les premiers projets et programmes dans un contexte de synergie des actions. L'option de la complémentarité entre projets et programmes est très positive, mais elle crée toutefois, une interdépendance qui peut être néfaste pour l'efficacité et la pérennité des interventions, si les projets dont dépendent les autres ne produisent pas les résultats attendus.

\subsection{Evaluation de la prise en compte de l'accessibilité des populations rurales aux services financiers dans la conception des projets et programmes de développement rural}

Tableau 6 : Analyse de l'accessibilité des populations rurales bénéficiaires des projets et programmes de développement rural aux services financiers

\begin{tabular}{|l|l|l|}
\hline Désignation & Nombre & Fréquence (\%) \\
\hline Très accessible & 18 & 5 \\
\hline Accessible & 53 & 16 \\
\hline Non accessible & 267 & 79 \\
\hline TOTAL & 338 & 100 \\
\hline
\end{tabular}

L'analyse du tableau 6 montre que les services financiers des établissements de microfinances selon les différentes parties prenantes sont non accessibles à $79 \%$, accessibles à $16 \%$ et très accessibles à seulement à $5 \%$. Donc, les projets et programmes de développement rural n'ont pas pris suffisamment en compte, les difficultés d'accès aux services financiers des populations rurales lors de leur conception, ainsi que de leurs mises en œuvre.

Le faible accès des populations rurales à des services financiers adaptés à leurs besoins est une contrainte majeure à leur épanouissement socioéconomique et culturel en milieu rural. L'accès aux services financiers doit permettre un meilleur accès aux intrants, matériels et équipements de production, de transformation etc. ainsi que permettre d'améliorer la résilience 
des ménages face aux situations imprévues (maladie, deuil, famine, etc.). Au Cameroun, l'accès des ruraux pauvres aux services financiers reste très limité car la non accessibilité est estimée à $79 \%$ selon les différents acteurs. Il est donc très indispensable que les projets et programmes soutiennent l'accès des ruraux pauvres à des services financiers adaptés à leurs besoins. Les projets et programmes (dans 20\% des cas) ont donné la priorité à l'amélioration des activités des Etablissements de Microfinance (EMF). C'est dans l'optique de faire l'extension de leurs domaines d'interventions pour la mise en place de produits financiers adaptés aux ruraux pauvres. Le volet de soutien à la demande de crédits et à l'utilisation desdits crédits, n'est pas suffisamment développé et la mise en œuvre s'est avérée complexe, rendant l'impact de crédits sur les revenus agricoles assez improbable, d'où rejet progressif par les producteurs et les OP de l'éventualité d'accès à des services financiers améliorés adaptés au secteur rural. C'est pour cela que, Ryckmans (1995) trouve que la nécessité de procurer des ressources monétaires peut faire passer pour irrationnelles certaines allocations du temps. Le besoin d'argent croît sans cesse : la monétarisation des rapports sociaux réduit les traditions et els échanges de travaux collectifs. Ainsi, l'accroissement des revenus est donc un objectif primordial. C'est à cet objectif même qu'est assujettie la participation des populations rurales à d'autres activités. Bon nombre de population rurale font un calcul rationnel de la rentabilité sociale et économique de leurs activités. Au travers de cette rentabilité, elles continuent de privilégier la cohésion sociale, celle du groupe familial et celle du ménage.

Pour l'accès aux services financiers, les projets et programmes ont dédié une importante partie de leurs ressources à la création de nouvelles caisses ou points de services et aux "innovations" des nouveaux produits financiers. Pourtant, les établissements de microfinances (EMF) opérant en zones rurales ne subissent pas de grande pression du marché et ne sont donc pas incités à créer de nouveaux points de services financiers ou d'introduire des nouveaux produits financiers innovants dans le domaine du crédit en milieu rural. Si ces interventions ont un coût additionnel et ont un risque plus élevé que les offres classiques, elles ne sont pas adoptées par les EMF. D'autre part, la majorité des EMF disposent d'une capacité limitée à s'étendre en raison, notamment, de services centraux défaillants ou fragilisés et subissent des coûts d'opération très élevés. En plus, les EMF non pas suffisamment le niveau d'expertise nécessaire pour développer de nouveaux services financiers adaptés aux divers besoins et contraintes des producteurs et OP en milieu rural selon les régions, les filières, le niveau d'intégration au marché etc.

Afin de faciliter l'accès durable aux services financiers de proximité et à un coût abordable, les projets et programmes auraient dû davantage travailler sur la réduction des coûts d'opération des EMF plutôt que sur la création de nouveaux points de services, nouveaux produits financiers complexes etc. 
Ainsi, ORSTOM-CIE, (1985), note qu'une connaissance précise des méthodes de production permet d'aider les populations rurales sans leur ôter le contrôle de leur activité. L'accès au crédit, comme moyen de pallier à la faiblesse des revenus, est une des solutions proposées aux populations rurales dans de nombreux projets/programmes. En l'absence de garantie par un titre foncier ou par du matériel agricole, ce sont les groupements de populations rurales ou des banques de populations rurales qui garantissent les emprunts individuels. L'extension géographique des points de services peut trouver sa faisabilité dans des aménagements de guichets mobiles ou grâce au recours de la téléphonie mobile pour faire baisser les coûts d'opération dans la mesure où la situation sécuritaire locale le permet.

Cependant, la mise en place du Fonds de facilitation basée sur l'hypothèse que le manque de ressources à moyen et long terme au niveau des EMF est la raison pour laquelle ceux-ci n'offraient pas de crédits moyen terme aux producteurs et $\mathrm{OP}$, alors que d'autres facteurs tels que les coûts d'opération élevés en zone rurale, expliquent la rareté des financements. Certains facteurs explicatifs sont : le manque d'expertise technique au sein des EMF pour analyser les dossiers de crédits relativement plus complexes ; la difficulté de mobiliser des garanties sûres ; la faible capacité des réseaux des producteurs et $\mathrm{OP}$, etc. En termes de gestion des risques, la capacité à absorber des crédits de plus gros montants en comparaison avec les crédits habituels octroyés et les risques encourus sur les ressources. Ces facteurs n'ont pas été suffisamment pris en compte lors de la conception des projets et programmes, d'où le faible nombre de crédits octroyés aux producteurs et OP par les EMF. Par contre, les études montrent un taux très élevé de recouvrement du crédit chez les populations rurales.

D’après Barrés, (1985), dans de très nombreux cas, les projets/programmes favorisant l'accès au crédit pour les populations rurales ont un impact positif à la fois sur les possibilités de production (intrants), de diversification de leurs activités et de l'alimentation de la famille. Les crédits sont aussi utilisés pour d'autres investissements dans la production ou pour permettre l'acquisition de biens de consommation. Pour Mottin-Sylla (1987), ces crédits, prenant le relais des tontines, permettent aux populations rurales de maintenir leurs réseaux de solidarité. Ils servent aussi aux dépenses « sociales » à l'occasion des fêtes religieuses ou familiales dont on connaît l'importance pour les populations rurales dans leurs stratégies d'alliance et pour le maintien des liens avec leur lignage. En allant dans le même sens, Odeye (1985) trouve que de plus en plus, de crédits sont accordés à titre individuel, avec une caution solidaire garantissant le contrôle social nécessaire au remboursement ; ils permettent des investissements à long terme dans des activités de transformation agricole ou de commercialisation. 


\subsection{Pertinence du ciblage social des projets et programmes de développement rural}

Tableau 7 : Appréciation de l'adéquation du ciblage social des projets et programmes de développement rural par les différentes parties prenantes

\begin{tabular}{|l|l|l|}
\hline Désignation & Nombre & Fréquence (\%) \\
\hline Adéquation du ciblage social & 289 & 86 \\
\hline Non adéquation du ciblage social & 49 & 14 \\
\hline TOTAL & 338 & 100 \\
\hline
\end{tabular}

L'analyse du tableau 7 montre que les enquêtés estiment que le ciblage social est en adéquation avec les besoins d'appuis à $86 \%$, par contre, $14 \%$ estiment que le ciblage n'est pas en adéquation avec les besoins des bénéficiaires. Ce qui veut dire que les ciblages sociaux des projets et programmes de développement sont pertinents et appropriés. Le bien-fondé du ciblage social des projets et programmes selon les différents acteurs, réside dans le fait qu'il permet d'atteindre une cible bien déterminée.

L'ensemble des documents de conception des projets et programmes mentionnent clairement que les groupes cibles sont les populations pauvres en milieu rural, mais ceux-ci peuvent appartenir à des catégories différentes. Les projets et programmes s'adressent surtout aux groupes sociaux particulièrement vulnérables en milieu rural. Les mécanismes de planification participatifs des projets et programmes au niveau local devaient s'assurer que ces populations soient atteintes, principalement les femmes et les jeunes ainsi que plus particulièrement, les couches les plus vulnérables, impliquées dans les filières choisies. Les projets et programmes ne disposent pas d'un mécanisme spécifique pour s'assurer que les populations particulièrement vulnérables en milieu rural puissent bénéficier des appuis. Les projets et programmes ne disposent pas de stratégies particulières pour assurer le ciblage. Les risques d'accaparement des bénéfices par des élites au sein des OP sont importants, mais aucune analyse ni suivi sont faits de ces phénomènes par les projets et programmes de développement rural à la quête des résultats.

Ryckmans (1995) estime que les projets/programmes ont pour objectif d'assurer aux populations rurales de nouvelles productions ou l'accès à de nouveaux marchés. Ces activités s'établissent dans le prolongement des activités habituelles des populations rurales ou des rôles qui leur sont traditionnellement confiés. L'objectif de ces projets/programmes est d'accroître les revenus des populations rurales, ce qui leur permet de pallier les effets des crises : la hausse des prix des produits manufacturés, la baisse des prix des produits agricoles, la sécheresse ou la désertification, les migrations, le coût croissant des services de santé ou d'éducation, le chômage, etc. 
Tableau 8 : Appréciation des différentes parties prenantes de l'atteinte de la cible social par les projets et programmes de développement rural

\begin{tabular}{|l|l|l|}
\hline Désignation & Nombre & Fréquence $(\%)$ \\
\hline Atteinte du ciblage social & 169 & 50 \\
\hline Non atteinte du ciblage social & 169 & 50 \\
\hline TOTAL & 338 & 100 \\
\hline
\end{tabular}

L'analyse du tableau 8 montre que le ciblage social est atteint à $50 \%$ et pas le cas dans $50 \%$ des circonstances. Ce qui veut dire que le ciblage dans la conception des projets/programmes comporte encore à $50 \%$ des manquements.

Les projets et programmes ciblent indirectement les bénéficiaires à travers les partenaires opérationnelles et des organisations rurales. Il est fort probable que les populations les plus vulnérables ne soient pas atteintes, du fait de ce ciblage indirect, combiné au fait qu'aucun effort n'ait fait pour adapter le ciblage aux conditions particulières de ces groupes lesquelles conditions qui sont parfois très complexes pour ces groupes vulnérables. Certaines caractéristiques du processus d'appuis des projets et programmes rendent difficile l'accès aux appuis à l'exemple de : la nécessité d'un patrimoine (terre, maison, etc.) pouvant servir de garantie pour accéder aux appuis ou aux crédits etc. Il est difficile de catégoriser les bénéficiaires en fonction de leurs revenus parce qu'ils dépendent majoritairement de leurs activités agropastorales et n'ont pas un accès direct à d'autres ressources. Ainsi, il existe un risque d'éloignement progressif de la cible première des projets et programmes. Dans les faits, quel que soit les projets et programmes, très peu ont été implémentés en s'assurant que les ressources et efforts soient prioritairement ciblés vers les couches les plus pauvres et vulnérables des populations rurales. Mais, cela nécessite selon Holvoet et Robrecht (2007), de définir à la fois les bénéficiaires des résultats et ceux qui expriment les cibles à atteindre.

La diversité ethnique au Cameroun n'est pas prise en compte lors de la conception des projets, la mise en œuvre ou dans les stratégies des partenaires techniques et financiers. Les analyses contextuelles effectuées ne considèrent pas la possible marginalisation de certains groupes ethniques comme étant l'une des causes de leur pauvreté et, par conséquent, ni les documents de conception, ni les rapports d'évaluation de la mise en œuvre des projets et programmes ne prévoient de ciblage spécifique à l'endroit de certains groupes ethniques considérés comme marginalisés, exception de $02 \%$ des projets et programmes. La problématique de la captation des ressources au profit d'une minorité, sur un fondement ethnique, social, linguistique, culturel ou politique est récurrente dans les projets et programmes de développement rural. Elle est d'autant plus exacerbée lorsque l'appui est véhiculé par des canaux indirects 
tels que les OP, qui peuvent être des enjeux de pouvoirs au niveau local. Toutefois, nous avons observé une forte concentration du pouvoir entre les mains de leaders politiques locaux au niveau de certaines OP appuyées par les projets et programmes en milieu rural. En outre, plusieurs évaluations des projets et programmes ont rapporté des phénomènes similaires d'accaparement par les élites locales. Toutefois, le niveau d'évidences disponibles ne permet pas de confirmer l'hypothèse d'une captation systématique des ressources par un groupe ethnique, social ou politique dominant des projets et programmes, ni d'éclairer les stratégies d'acteurs au niveau local.

Cependant, Frisa (2019) trouve qu'un manque de coordination et de légitimité, une distribution asymétrique de l'information entrainent une capture des fonds par les élites. En effet, en zone rurale, les individus appartiennent à des communautés héritées (familles, clans, tribus) au sein desquelles sont entretenus des liens forts, des liens de sang et qui partagent des valeurs, des idéologies et des règles partagées et intériorisées depuis des siècles. Ce contexte donne une forte légitimité aux représentants des communautés, soit aux Chefs de famille, aux Chefs de village, aux Chefs de groupement qui héritent de leurs fonctions. Dans une société où la pauvreté règne, il est clair que les financements obtenus auprès des partenaires internationaux permettent aux organisations de réaliser des actions mais ne permettent pas forcément aux citoyens d'exprimer leurs besoins, ni aux organisations de monter des projets correspondants aux besoins des populations.

\subsection{Pertinence du ciblage géographique et considérations politiques des projets et programmes de développement rural au Cameroun}

La carte des projets financés par les partenaires techniques et financiers au Cameroun met en évidence une couverture intégrale (100\%) du territoire camerounais par les projets depuis 2000 à 2018. La stratégie de ciblage géographique des projets et programmes au Cameroun est théoriquement focalisée sur l'incidence de la pauvreté dans les dix (10) régions du pays, mais aussi sur des considérations d'ordre politique. Suite au ciblage plus qu'ambitieux des projets et programmes, dont la couverture géographique est nationale, les partenaires techniques et financiers préconisent surtout une localisation centrée autour des régions les plus pauvres que sont le Nord, l'Est, l'Extrême-Nord et le Nord-Ouest. C'est ainsi que les projets et programmes couvrent principalement de plus en plus ces régions, mais aussi l'Ouest et le Centre. Le choix préalable de ces régions a d'ailleurs pu être déterminant dans le choix des filières appuyées par les projets et programmes. Le ciblage des régions anglophones (notamment le Nord-Ouest), présent dans quasiment tous les projets et programmes, s'inscrit aussi dans une volonté du Gouvernement d'orienter les financements vers des zones à forte sensibilité politique. 
Bien que les stratégies des partenaires techniques et financiers optent pour une concentration géographique dans ces régions suscitées des interventions dans l'optique de la consolidation des acquis des projets/programmes antérieurs, le ciblage des autres régions par les projets/programmes, contribue davantage à la dispersion des interventions des partenaires techniques et financiers sur le plan national. Cette dispersion ne favorise pas le renforcement de la complémentarité des projets/programmes pourtant souhaité dans les orientations stratégiques. De plus, les régions du Littoral et du Centre sont parmi les régions les moins pauvres et où la pauvreté est en baisse comparée aux autres régions, même si les données agrégées du niveau de pauvreté cachent des inégalités parfois importantes au sein des populations rurales des dix (10) régions du pays.

\subsection{Insuffisance de la prise en compte de la gestion des risques dans la conception des projets et programmes de développement rural}

Tableau 9 : Appréciation de la prise en compte des risques des projets et programmes de développement rural

\begin{tabular}{|l|l|l|}
\hline Modalités & Nombre & Fréquence (\%) \\
\hline Prise en compte & 82 & 24 \\
\hline Modérément prise en compte & 67 & 20 \\
\hline Pas pris en compte & 189 & 56 \\
\hline TOTAL & 338 & 100 \\
\hline
\end{tabular}

L'analyse du tableau 9 montre que dans la conception des projets/programmes, la prise en compte des risques est de $24 \%$, avec $20 \%$ de prise en compte de manière modérée et $56 \%$ de non prise en compte. Ce qui veut dire que les projets et programmes de développement ne prennent suffisamment en compte la gestion des risques.

Les documents de conception des projets et programmes sont généralement élaborés de manière optimiste et se fondent sur la conviction que les appuis et investissements seront réalisés. Néanmoins, la faible performance des projets et programmes de développement rural au Cameroun, amènent les concepteurs à mener des réflexions sur les risques et les facteurs d'atténuation de leurs impacts. Les risques recensés dans les documents de conception des projets et programmes concernent principalement les aspects techniques, qui ne se sont pas vraiment des points critiques. Un véritable risque critique de la plupart des projets/programmes est celui des retards dans leur mise en œuvre également mentionné les documents de conception des projets et programmes, mais les mesures d'atténuation y relatives suggérées s'avèrent ne pas être pertinentes. Les risques liés aux résultats attendus d'autres projets et programmes dans le cadre de la complémentarité et des synergies des projets et programmes de développement rural ne sont pas souvent bien analysés. 
Il ressort globalement de l'évaluation des risques que ceux liés à une mauvaise gouvernance et à des mauvais systèmes pour garantir l'efficience des projets et programmes lors de leur mise en œuvre ne sont pas suffisamment pris en compte. Les risques recensés dans les projets et programmes examinés sont qualifiés d'être élevés avec des mesures d'atténuation limitées. La FAO (2014) estime d'ailleurs que les investissements des donateurs ou du gouvernement obéissent à une stratégie prédéterminée (visant par exemple à accroître la production agricole, la productivité et les revenus des exploitants). L'unité de gestion du projet et les instances de mise en œuvre ont tendance à intervenir du haut vers la base. Dès les premières étapes de l'identification du projet, il faut déterminer dans quelle mesure les bénéficiaires visés peuvent accepter et adopter les technologies et les services associés.

Le risque de retards de mobilisation des fonds de contrepartie pour la mise en œuvre des projets et programmes identifié, est pratiquement systématique dans tous les portefeuilles des partenaires techniques et financiers. La mise en œuvre des projets et programmes rencontre généralement trop de difficultés à cause des retards de la mise à disposition (et, aussi de la réduction des montants) des fonds de contrepartie. La pertinence globale moyenne des projets et programmes qui ont fait l'objet de la recherche est jugée modérément insatisfaisante.

Ceci nous a amené à faire ressortir les tendances de l'évolution de la pertinence des projets et programmes de développement rural au Cameroun durant les deux dernières décennies. 
3.10. Evaluation de l'évolution de la pertinence des projets et programmes de développement rural dans la conception et la mise en ouvre

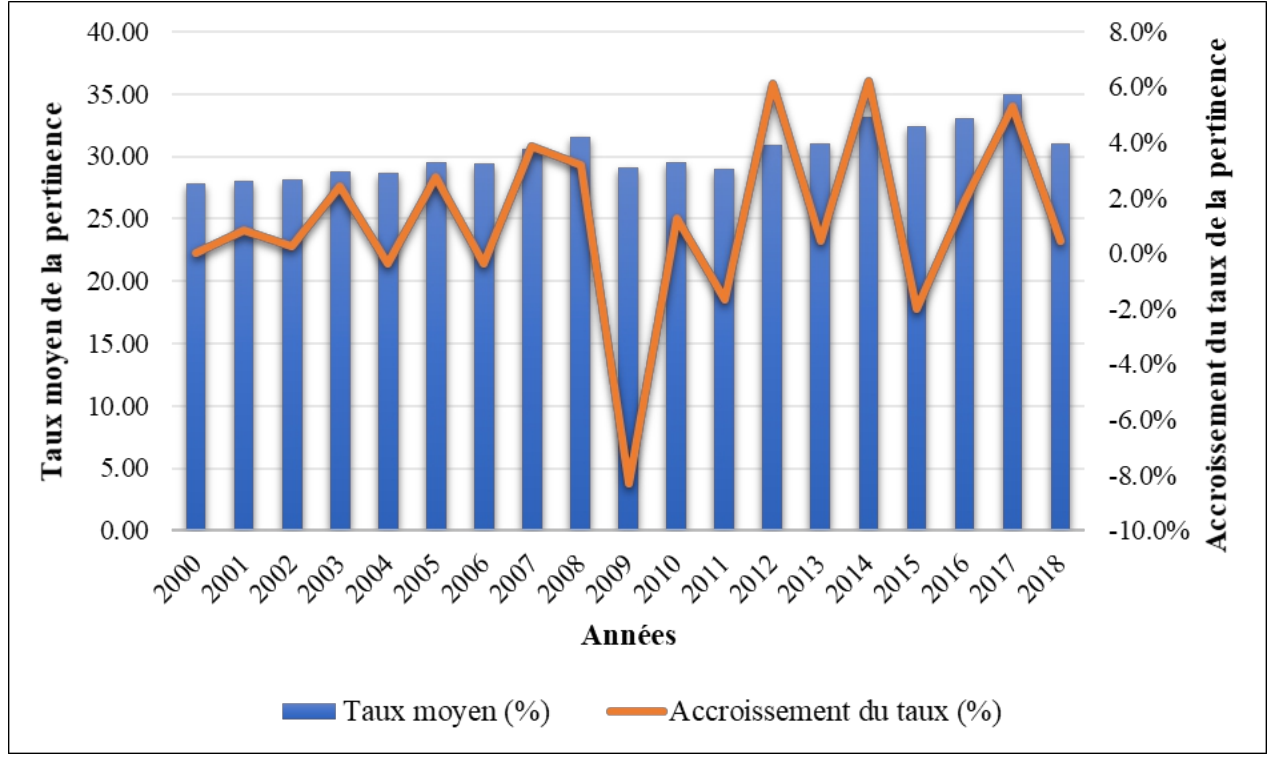

Figure 3 : Evolution de la pertinence des projets et programmes de développement rural dans leur conception et leur mise en œuvre

La figure 3, représente les tendances de l'évolution du taux moyen de la pertinence et de l'accroissement de ce taux moyen dans la conception et la mise en œuvre des projets et programmes de développement rural durant la période de 2000 à 2018. Le taux moyen de la pertinence étant représenté en barres bleus tandis que la courbe de l'accroissement des taux est représentée en courbe orange.

Le taux moyen de la pertinence des projets et programmes de développement rural dans leur conception et leur mise en œuvre le plus bas est de $27,78 \%$ en 2000, tant dis que le taux moyen le plus élevé est de 34,93\% enregistré en 2017. Le sens d'évolution des taux moyens n'est pas très variable de manière générale et la tendance reste presque à la hausse. Cependant, il est à noter que la moyenne des taux moyen globaux de la pertinence est de $30,34 \%$. Ce résultat concorde avec la pertinence globale moyenne des cinquante-six (56) projets et programmes de notre étude qui est jugée modérément insatisfaisante.

La courbe d'accroissement des taux de la pertinence des projets et programmes de développement rural lors de leur conception et leur mise en œuvre évolue en zigzag mais la tendance est à la hausse de manière globale. Par ailleurs, nous constatons que le niveau d'accroissement le plus bas est de $-8,31 \%$ enregistré en 2009 , tans dis que, le niveau d'accroissement le plus élevé enregistré en 2017 est de 20,45\%. Par ailleurs, il est à noter que nous 
enregistrons également une baisse de l'accroissement du taux de la pertinence en 2018 qui est de 10,50\%, comparé aux accroissements de 2013 qui est à 10,50\% et la tendance reste croissante jusqu'à atteindre 20,45\% en 2017. Cependant, il est à noter que la moyenne de l'accroissement du taux global est de $8,07 \%$. Selon les résultats de l'étude, le taux d'accroissement corrobore avec la pertinence globale moyenne des projets et programmes de développement rural au Cameroun qui est jugée modérément satisfaisante. Les projets et programmes de développement rural au Cameroun ne sont pas pertinents dans leurs conceptions et leurs mises en œuvre. Il serait nécessaire, voir impératif de repenser les stratégies et les approches de conception et de mise en œuvre des projets et programmes de développement rural au Cameroun.

\subsection{Corrélations entre la pertinence financière et la pertinence physique des projets et programmes de développement rural}

Tableau 10 : Corrélations

\begin{tabular}{|c|c|c|c|c|c|c|c|}
\hline & $\begin{array}{l}\text { Montant du } \\
\text { financement }\end{array}$ & $\begin{array}{l}\text { Montant } \\
\text { décaissé }\end{array}$ & $\begin{array}{l}\text { Performanc } \\
\text { e financière }\end{array}$ & $\begin{array}{l}\text { Performan } \\
\text { ce } \\
\text { physique }\end{array}$ & $\begin{array}{l}\text { Taux } \\
\text { moyen } \\
(\%) \\
\end{array}$ & $\begin{array}{l}\text { Accroisseme } \\
\text { nt du taux } \\
(\%)\end{array}$ & $\begin{array}{l}\text { Perti } \\
\text { nenc } \\
\mathrm{e}\end{array}$ \\
\hline $\begin{array}{l}\text { Montant du } \\
\text { financement }\end{array}$ & $1 * *$ & & & & & & \\
\hline $\begin{array}{l}\text { Montant } \\
\text { décaissé } \\
\text { Performance }\end{array}$ & $0,656^{*}$ & $1 * *$ & & & & & \\
\hline financière & $-0,3552$ & 0,4689 & $1 * *$ & & & & \\
\hline physique & $-0,3090$ & $0,506^{*}$ & $0,987 * *$ & $1 * *$ & & & \\
\hline $\begin{array}{l}\text { Taux moyen } \\
(\%)\end{array}$ & $0,592 *$ & 0,2595 & $-0,3352$ & $-0,2965$ & $1 * *$ & & \\
\hline $\begin{array}{l}\text { Accroisseme } \\
\text { nt du taux } \\
(\%)\end{array}$ & 0,0101 & $-0,0849$ & $-0,0802$ & $-0,1031$ & 0,4596 & $1 * *$ & \\
\hline Pertinence & 0,1222 & 0,2534 & 0,1866 & 0,1712 & 0,0836 & $-0,3416$ & $1 * *$ \\
\hline
\end{tabular}

D'après la matrice de corrélation du tableau 10 , il ressort qu'il y'a une corrélation significative modérée entre le montant du financement et le montant décaissé $\left(\mathrm{r}=0,65^{*}\right)$. Lorsque le montant du financement augmente, le montant décaissé augmente aussi modérément. Il existe aussi une corrélation significative modérée entre le montant du financement et le taux moyen de la pertinence des projets et programmes de développement rural dans leur conception et leur mise en œuvre $(\mathrm{r}=0,59 *)$. Lorsque le montant du financement augmente, le taux moyen de la pertinence augmente aussi.

Dans le cas où $r$ est strictement inférieur à 0,5 , il existe une relation faible corrélation; au fur et à mesure que le coefficient s'approche de 0 , la corrélation est non significative c'est-à-dire qu'il n'y a pas corrélation. Il 
ressort du tableau 12 qu'il n'y a pas corrélation entre le montant du financement et la performance financière où $(r=-0,35)$. Il n'existe pas de corrélation entre le montant du financement et la performance physique où $(\mathrm{r}=-0,30)$. Il n'y a pas de corrélation entre le montant du financement et l'accroissement du taux de la pertinence des projets et programmes de développement rural dans leur conception et leur mise en œuvre où $(r=0,26)$.

Du même tableau, il est à noter qu'il n'existe pas de corrélation entre le montant décaissé et la performance financière où $(\mathrm{r}=0,46)$. Par contre, une corrélation significative modérée existe entre le montant décaissé et la performance physique $(\mathrm{r}=0,50 *)$. Lorsque le montant décaissé augmente, la performance physique augmente aussi modérément. Il n'y a pas corrélation entre le montant décaissé et le taux moyen de la pertinence par rapport à la conception et à la mise en œuvre des projets et programmes de développement rural où $(\mathrm{r}=0,24)$. Il n'y a pas de corrélation entre le montant décaissé et l'accroissement du taux moyen de la pertinence des projets et programmes de développement rural dans leur conception et leur mise en œuvre où $(r=0,30)$.

Dans le cas où le coefficient $r$ est compris entre 0,8 et 1 , il $\mathrm{y}$ a une corrélation significativement forte. C'est le cas de la relation entre la performance financière et la performance physique où $\left(\mathrm{r}=0,98^{* *}\right)$, ce qui veut dire qu'il y' a une forte corrélation significative entre la performance financière et la performance physique. Lorsque la performance financière augmente, la performance physique augmente aussi. Toutefois, nous constatons qu'il n'y a pas corrélation entre la performance financière et le taux moyen de la pertinence des projets et programmes de développement rural dans leur conception et leur mise en œuvre où $(r=-0,33)$. Il n'y a pas corrélation entre la performance financière et l'accroissement du taux moyen de la pertinence des projets et programmes de développement rural dans leur conception et leur mise en œuvre où $(\mathrm{r}=0,12)$.

Il n'y a pas corrélation entre la performance physique et le taux moyen de la pertinence des projets et programmes de développement rural dans leur conception et leur mise en œuvre où $(\mathrm{r}=-0,30)$. Il n'y a pas de corrélation entre la performance physique et l'accroissement du taux moyen de la pertinence des projets et programmes de développement rural dans leur conception et leur mise en œuvre où $(\mathrm{r}=0,09)$. Nous trouvons qu'il n'y a pas de corrélation entre le taux moyen de la pertinence et l'accroissement du taux moyen de la pertinence des projets et programmes de développement rural dans leur conception et leur mise en œuvre où $(r=0,49)$.

Il n'y a pas corrélation entre la pertinence et le montant du financement $(\mathrm{r}=0,12)$. Il n'y a pas corrélation entre la pertinence et le montant décaissé $(\mathrm{r}=0,25)$. Il n'y a pas corrélation entre la pertinence et la performance physique des projets et programmes de développement rural dans leur conception et leur mise en œuvre $(\mathrm{r}=0,18)$. Il n'y a pas corrélation entre la pertinence et la 
performance financière de la pertinence des projets et programmes de développement rural dans leur conception et leur mise en œuvre $(\mathrm{r}=0,17)$. Il n'y a pas corrélation entre la pertinence et le taux moyen de la pertinence des projets et programmes de développement rural dans leur conception et leur mise en œuvre $(\mathrm{r}=0,08)$. Il n'y a pas corrélation entre la pertinence et l'accroissement du taux moyen de la pertinence des projets et programmes de développement rural dans leur conception et leur mise en œuvre $(\mathrm{r}=-0,34)$.

\section{Conclusion}

La pertinence des projets et programmes de développement rural au Cameroun, a été analysée via l'examen de l'ensemble des résultats de la conception et de la mise en œuvre desdits projets et programmes de développement rural. Au terme de cet évaluation, il convient de retenir que : les projets et programmes de développement rural ont une pertinence globale moyenne modérément insatisfaisante. A ce titre, l'orientation des résultats des projets et programmes sur les plans technique et financier constitue un goulot d'étranglement. La contribution des projets et programmes de développement rural rapportée aux pertinences, rend impossible le jugement appréciatif de la valeur ajoutée de ces projets et programme de développement rural de manière tranchée.

En effet, les principaux résultats enregistrés à l'issu de cette première évaluation de la pertinence des projets et programmes de développement rural sur le plan national sont intéressant malgré que cette pertinence soit modérément insatisfaisante. Au regard des variables explicatives de la pertinence globale des projets et programmes de développement rural, nos résultats se résument dans le sens de l'atteinte des objectifs initiaux de conception et de mise en œuvre non satisfaisants. Ceci dit, la pertinence de ces projets et programmes est perfectible sous réserve de l'intégration des éléments d'amélioration.

\section{Remerciements}

Ces travaux ont été réalisés dans le cadre de la recherche d'une thèse de doctorat/PhD. Les auteurs tiennent aussi à remercier toutes les parties prenantes qui ont contribué, de manière directe ou indirecte, à la réalisation de la présente étude.

\section{References :}

1. Abega A. R. (2015). La performance managériale dans les infrastructures au Cameroun : une approche par le suivi-évaluation. Mémoire Online. Université Internationale Senghor-Master 2 en Développement, spécialité Management des projets. 47 pages. 
2. Banque Mondiale (2006). Rapport annuel. Bureau des publications, Relations extérieures. The International Bank for Reconstruction and Development / The World Bank. 1818 H Street N.W. Washington D.C. 20433. Site web : www.worldbank.org. 66 pages.

3. Banque Mondiale (2007). "Rapport sur le développement dans le monde 2008 : l'agriculture au service du développement » Résumé publié en octobre 2007. The International Bankfor Reconstruction and Development / The World Bank. 1818 H Street, NW Washington, DC 20433, États-Unis d'Amérique. 36 pages.

4. Banque Mondiale (2008). «Rapport sur le développement dans le monde. L'agriculture au service du développement », Mondes en développement 3/2008 ( $\mathrm{n}^{\circ}$ 143), p. 117-136 URL : www.cairn.info/revue-mondes-en-developpement-2008-3-page117.htm. 68 pages.

5. Cahiers économiques du Cameroun (2011). Janvier 2011. Numéro 1. Le réveil du lion ? Point sur la situation économique du Cameroun. Spécial télécommunications. Unité de la réduction de la pauvreté et la gestion économique, région Afrique. 23 pages.

6. Document de Stratégie pour la Croissance et l'Emploi (2009). Stratégie pour la Croissance et l'Emploi. 167 pages. https://paris21.org/sites/default/files/Cameroon DSCE2010-20.pdf

7. ELA J-M. (1990). Quand l'Etat pénètre la brousse : la riposte des paysans à la crise. Karthala, Paris, 268 pages.

8. Fonds International de Développement Agricole (2017). Perspectives : le FIDA dans le contexte du Programme de développement durable à l'horizon 2030. Consultation sur la Onzième reconstitution des ressources du FIDA. Deuxième session. Rome, 29 - 30juin 2017. IFAD11/2/R.2. 34 pages.

9. Fontil (2009). Projet de développement communautaire en Haïti : Méthodologie d'analyse des besoins locaux. Mémoire présenté pour l'obtention du Master en Développement de l'Université Senghor. Département Administration-Gestion. Spécialité Management de Projet. 363 pages.

10. Institut National de la Statistique (2015). Quatrième enquête camerounaise auprès des ménages (ECAM 4). Tendances, profil et déterminants de la pauvreté au Cameroun entre 2011-2014. Boîte Postale : 134 Yaoundé. 65 pages.

11. Institut National de la Statistique (2010). Annuaire Statistique du Cameroun 2010. Deuxième Partie : Population et Affaires sociales. 14 pages.

12. Kamtchouing N. P. (2009). La pratique du suivi évaluation dans les projets de développement au Cameroun. - Pour l'obtention du Master 
en Développement (Management de Projets) de l'Université Senghor d'Alexandrie. Département Administration - Gestion. Spécialité Management de projets, le 22 avril 2009. Mémoire Online. 62 pages.

13. Merlin P. (1991). Espoir pour l'Afrique Noire. Présence africaine, Paris, 96 pages.

14. Organisation de Coopération et de Développement Economiques/Food and Agriculture Organization of the United Nations (2016). L'agriculture en Afrique subsaharienne : Perspectives et enjeux de la décennie à venir PARTIE I. Chapitre 2. Perspectives agricoles de l'OCDE et de la FAO 2016-2025. 43 pages. 